\title{
Current Controversies in Large-Vessel Inflammatory Vasculitis and Thoracic Aortic Aneurysm Disease
}

\author{
Amer Harky, MRCS ${ }^{1}$ Matthew Fok, MRCS ${ }^{2}$ Callum Howard, BSc ${ }^{3}$ Mohamad Bashir, MD, PhD, MRCS 3 \\ ${ }^{1}$ Department of Vascular Surgery, Countess of Chester Hospital, \\ Chester, United Kingdom \\ 2 Department of General Surgery, Royal Liverpool Hospital, Liverpool, \\ United Kingdom \\ ${ }^{3}$ Department of Aortovascular Surgery, Manchester Royal Infirmary, \\ Manchester, United Kingdom \\ Address for correspondence Mohamad Bashir, MD, PhD, MRCS, \\ Department of Aortovascular Surgery, Manchester Royal Infirmary, \\ Manchester, United Kingdom (e-mail: drmbashir@mail.com). \\ Int J Angiol 2019;28:215-225.
}

\begin{abstract}
Keywords

- large vessel

- vasculitis

- thoracic aortic disease

Large-vessel vasculitis encompasses the spectrum of vasculitides, which pathologically cause chronic granulomatous inflammatory changes, primarily in the aorta and its major branches. These patients are at risk of developing life-threatening aortic lesions that, without recognition and prompt treatment, can cause detrimental effects. Many provocative issues surrounding large-vessel vasculitis and its surgical treatment still remain, spanning from recognition to management. In this review, we discuss the main large-vessel vasculitides, Takayasu's arteritis and giant cell arteritis. We include the key points and current controversies surrounding diagnostic imaging, timing of interventions, and patient outcomes.
\end{abstract}

Large-vessel vasculitis (LVV) encompasses the spectrum of vasculitides, which cause pathological chronic granulomatous inflammatory changes, primarily in the aorta and its major branches. Takayasu's arteritis (TA) and giant cell arteritis (GCA) form two major entities of this spectrum that can potentially affect the aorta ( - Fig. 1). Today, many consider TA and GCA to be a spectrum of the same disease with a bimodal age of onset. There are few published studies that elucidate when patients with these large-vessel vasculitides should be evaluated for and undergo thoracic aortic surgery, partly because few patients ultimately require surgical treatment. Few studies have examined when patients with LVV should undergo surgical treatment. There are, however, many important surgical issues in the management of these patients. Patients with TA are usually younger. In contrast, patients with GCA are often older than 75 years, posing additional challenges. In particular, the diagnosis, medical treatment, and timing of these surgical interventions require close involvement of the surgeon as part of a multidisciplinary team and lifelong follow-up. The areas of controversy currently focus upon the optimal imaging for these patients, surgical management, and medical management. This review will give an overview of the two major largevessel vasculitides, namely, TA and GCA, the role of IgG4 syndrome, and an introduction to the disagreements in their management.

\section{Clinical Presentation of Takayasu's Arteritis and Giant Cell Arteritis}

\section{Clinical Presentation of Takayasu's Arteritis}

TA, historically known as "pulseless disease," was first identified in 1908 by the Japanese ophthalmologist Mikito Takayasu and later described by renowned surgeons Kentaro Shimizu and Keiji Sano in 1951. 2,3 However, TA's significance in terms of large-vessel pathology was not fully comprehended until a case report in 1940s came out, when the associated ophthalmic lesions were discovered to be secondary to panarteritis of the aorta. 4

Clinically, TA is thought to occur in two phases with varying predominating symptoms. In the systemic phase, patients present with symptoms of active inflammatory illness, such as fever, fatigue, weight loss, arthritis, and generalized aches and pains. Some patients may demonstrate tenderness associated with the affected arteries. Increased erythrocyte sedimentation rate (ESR) is a common finding during this phase. The second phase is the occlusive 


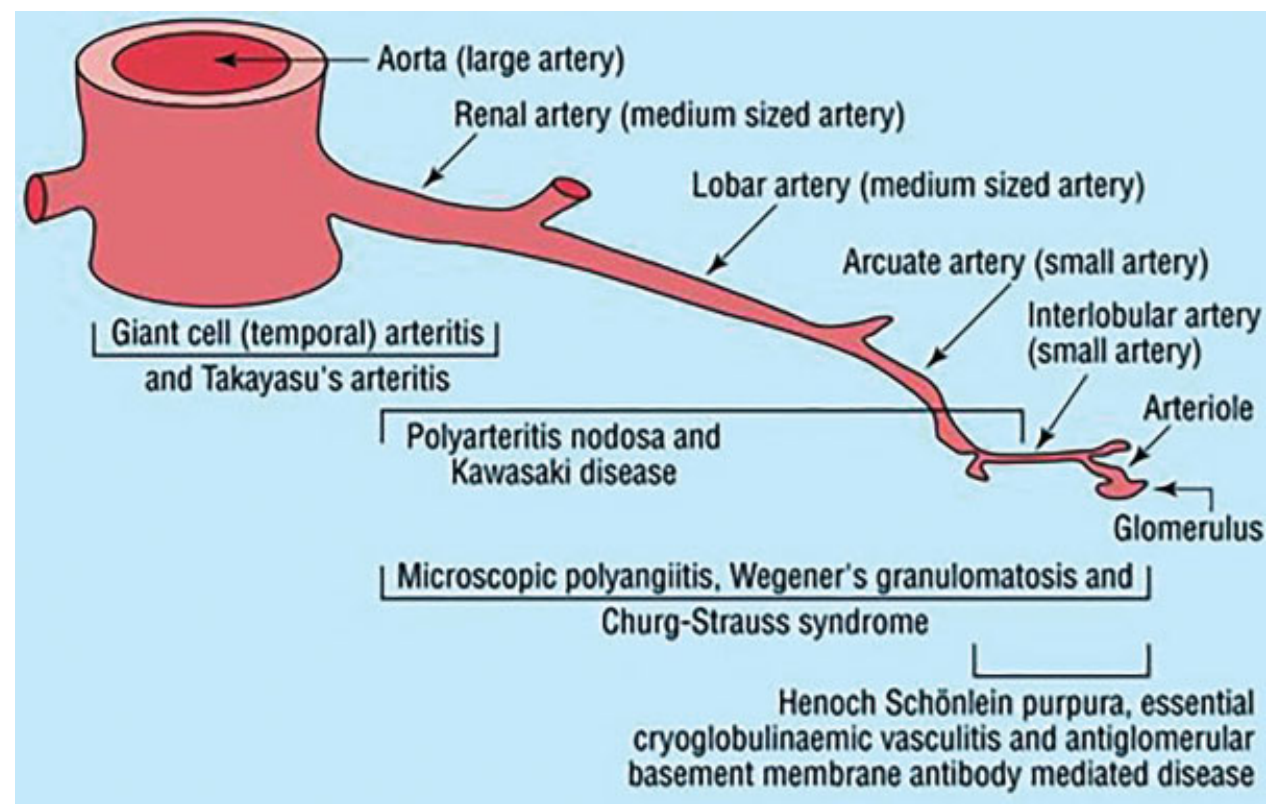

Fig. 1 From Savage et al ${ }^{1}$ Chapel Hill Consensus Criteria of vasculitis which displays the spectrum of systemic vasculitides and the involvement according to vessel size with permission.

phase, which presents with symptoms of stenosis in affected arteries. Symptoms may include claudication of the muscle groups receiving blood from the affected vessels, dizziness, headaches, and visual disturbances. As described historically, pulses may not be perceived in stenotic arteries, and bruits may be appreciated. High blood pressure is common; however, blood pressure may be falsely lowered if the stenosis occurs in the more proximal vessels. It is in this occlusive phase that retinal vessel malformations occur, as originally described.

In general, TA typically presents in female patients younger than 40 years and predominantly affects the aorta and its major branches. This pathology presents according to five general patterns, which range from aortitis of the entire aorta to only affecting one or more of the supra-aortic vessels. $^{5}$

The topographical classification of aneurysm formation in Takayasu's was developed in 1967 and revised in 1994, now including five types of TA. ${ }^{6}$ Historically, only the aortic arch and its branches were thought to be involved; however, improved imaging modalities and an increase in published reports of TA revealed that this is the case in only $8.4 \%$ of patients ( - Fig. 2, type 1 and 1 a). ${ }^{8}$ Type 2 a refers to lesions affecting the descending and abdominal aorta, which corresponds to $\sim 11.2 \%$ of cases, while type $2 \mathrm{~b}$ also includes the thoracic descending aorta ( - Fig. 2, type 2 ). Type 3 , by far the most common presentation in TA, and affects both the thoracic and abdominal aorta distal to the arch in $65.4 \%$ of patients (-Fig. 2, type 3). Type 4 refers to patients in whom the pulmonary artery is involved and occurs in $\sim 15 \%$ of patients ( - Fig. 2, type 4). Finally, type 5 includes the combined features of types 4 and $2 \mathrm{~b}$ (-Fig. 2, type 5). The aortic arch is most likely affected by panarteritis, resulting in stenosis or potential obstruction of the affected vessels. Most commonly, TA lesions involve the common carotid and subclavian arteries. Thus, due to the location of the aortic arch, any surgical repair to this area involves the inherent risk of damage to nearby critical structures. ${ }^{9}$

\section{Clinical Presentation of Giant Cell Arteritis}

GCA is a relatively new definition in terms of classification and diagnosis. Due to its comorbidity with polymyalgia rheumatica, GCA was not always seen as a separate disease process from TA. Now, it has been shown that GCA is a separate inflammatory condition and the most common primary vasculitis in adults. GCA has been historically associated with the temporal artery; however, GCA is not limited to the temporal artery and can affect a multitude of vessels, including the aorta.

When compared with TA, GCA generally presents in older patients, yet similarly still affects women more often than men. The most common symptoms of GCA are headache, mandibular claudication, fever, and blurred vision. ${ }^{10}$ Sometimes pain also occurs in the shoulders and hips, necessitating the importance of differentiating GCA from polymyalgia rheumatica. Other symptoms may include scalp tenderness, cough, odynophagia, weight loss, depression, or stroke. ${ }^{11,12}$ Similar to the presentation of TA in the occlusive phase, some patients experience extremity claudication.

Because the clinical presentation of GCA is varied, early diagnosis requires a heightened suspicion by clinicians. Not only is the aorta is very similarly affected in GCA as it is in TA, but also current timing for surgical intervention has not been identified in a disease-specific way.

\section{Epidemiology of Takayasu's Arteritis}

TA is rare with an estimated incidence of 1 to 2 per million inhabitants in Western countries. ${ }^{13}$ The rarity of TA is demonstrated in the lack of consistent epidemiological data available in published literature. European estimates 


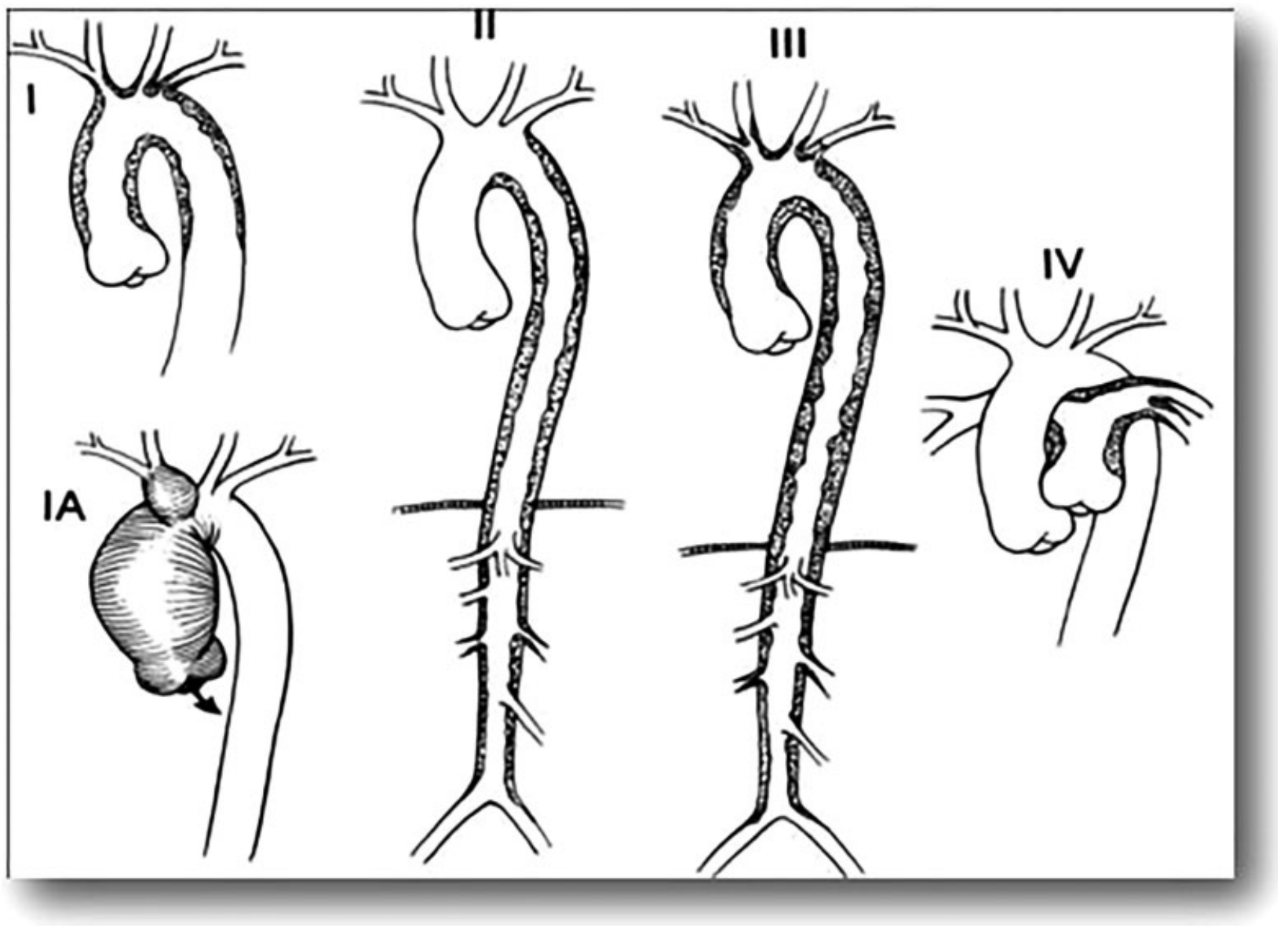

Fig. 2 The topographical classification of aneurysm formation in Takayasu's arteritis with permission. ${ }^{7}$

have shown incidences of 0.4 to 0.8 per million inhabitants in the United Kingdom, 0.5 per million in Germany, and 0.8 per million in Sweden. ${ }^{14-16}$ Estimates made from a Scandinavian population in Minnesota, United States, reported an incidence of 2.6 per million. ${ }^{17}$ The vast majority of TA cases originate from Japan, reflecting a reported 100 times higher incidence in Eastern Asia compared with Europe and the United States. There is a predominance of women who suffer TA, Koide et al reported a ratio of 8-9:1 in Japan, ${ }^{18}$ and peak age of onset is commonly 15 to 30 years. ${ }^{19,20}$

\section{Epidemiology of Giant Cell Arteritis}

The true incidence of GCA is unknown, with incidences ranging from 17.8 per 100,000 persons older than 50 years $^{21}$ to incidence reports ranging from 0.49 to 27.3 per 100,000 in the United States. ${ }^{22}$ The incidence of GCA is positively associated with increasing latitude, making GCA more common in Scandinavian countries. ${ }^{23}$ Moreover, aging is the greatest risk factor for developing GCA; it rarely occurs before the age of 50 years. ${ }^{7}$ While GCA predominates in the Caucasian population, the disease also displays a strong predilection for women, occurring two times more frequently in females, with a peak incidence of 60 to 80 years of age. ${ }^{10}$

Further, there is ample evidence for varying incidences of GCA among different ethnic groups, with Caucasian populations from Northern Europe and of Northern European descent being the most affected. ${ }^{24}$ One retrospective review conducted in California and spanning 12 years found that, when controlled for race, Caucasian patients had a significantly increased incidence of a GCA-positive biopsy. Based on biopsy alone, positive samples were demonstrated in $29 \%$ of Caucasian patients, compared with $11 \%$ in Asian and $0 \%$ in Hispanic and African American patients. ${ }^{25}$

GCA predisposes patients to related comorbidities and mortality. In 1995, Evans et al conducted a population-based cohort study of 96 residents from Minnesota, a population of Scandinavian descent, who were diagnosed with GCA. ${ }^{26}$ Of the participants, $11 \%$ developed a thoracic aortic aneurysm which equated to patients with GCA to be 17.3 more times likely to develop a thoracic aortic aneurysm. A current retrospective analysis of GCA shows that development of aneurysmal disease takes years after diagnosis to be established. Conversely, aortic dissection is often seen in the earlier stages of the disease that may not be attributed to aortic size.

Furthermore, untreated GCA can lead to permanent visual loss, occurring in an estimated $15 \%$ to $20 \%$ of GCA patients, and as such is a neuro-ophthalmic emergency. ${ }^{27}$

\section{Pathogenesis of Takayasu's Arteritis}

To date, the underlying pathogenesis of TA has not been completely understood; there are several different concepts proposed to understand the pathogenesis. Although current concepts suggest that the pathogenesis stems from $T$ lymphocytes, more specifically, gammadelta $(\gamma \delta)$ lymphocytes, 
cytotoxic T lymphocytes, T helper cells, current literature also supports that $\mathrm{B}$ lymphocytes and macrophages mediate the process. This is supported by histological evidence of a granulomatous panarteritis with cell wall infiltration of the vessel. $^{1,28,29}$ A key activator in the inflammatory process seems to be the expression of the $65 \mathrm{kDa}$ heat-shock protein in the aortic tissue; however, it is unclear as to what initiates the heat-shock protein. The heat-shock proteins induce the major histocompatibility class I chain-related A (MICA) on vascular smooth muscle, which is recognized by $\gamma \delta \mathrm{T}$ cells and NK cells. These cells subsequently release perforin, triggering vascular inflammation. Furthermore, research demonstrates the activation of dendritic cells through major histocompatibility complex alleles with toll-like receptors (TLRs) on T cells. Meanwhile, Th1 lymphocytes drive the formation of giant cells and activate macrophages with release of VEGF and PDGF, resulting in neovascularization and intimal proliferation. The exact role of dendritic cells in TA is highly debated, although one theory suggests that dendritic cells with B lymphocytes trigger an autoimmune reaction, resulting in complement-dependent cytotoxicity against endothelial cells. Further research into the initiation of these pathways may offer a better understanding in how to manage these patients. Ultimately, successful medical management may decrease the need for surgical treatment in the distant future.

\section{Pathogenesis Giant Cell Arteritis}

There are many similarities between the inflammatory pathogenesis of TA and GCA. Furthermore, also like TA, the exact pathogenesis underpinning GCA still remains elusive. As expected of a granulomatous infiltrate, the cells that make up the inflammatory response include $\mathrm{T}$ lymphocytes, macrophages, dendritic cells, multinucleated giant cells, and fibroblasts. ${ }^{30}$ Similar to TA, both innate and adaptive immune systems ultimately drive local vascular damage, leading to intimal hyperplasia, and eventually luminal stenosis and occlusion. ${ }^{31,32}$ However, there seems to be greater evidence for the involvement of dendritic cells in the process of GCA. Recent research has demonstrated the activation of resident adventitial dendritic cells via TLRs, specifically TLRs 2 and 4 . Once activated, dendritic cells activate the differentiation and recruitment of T cells, which produce IFN- $\gamma$, a key driver of vascular inflammation in GCA. ${ }^{33}$

\section{Diagnostic Criteria of Takayasu's Arteritis}

There are two diagnostic criteria specifically used for TA (-Tables 1 and 2). ${ }^{34}$ In the American College of Rheumatology classification criteria, the presence of 3 or more criteria
Table 2 Ishikawa: proposed criteria for the clinical diagnosis of Takayasu's arteriopathy

Obligatory criterion:

- Age less than or equal to 40 years

Major criteria:

- Lesion of the left mid subclavian artery

- Lesion of the right mid subclavian artery

Minor criteria:

- High ESR

- Common carotid artery tenderness

- Hypertension

- Aortic regurgitation or annuloaortic ectasia

- Lesions of the pulmonary artery

- Lesions of the left mid common carotid artery

- Lesions of the distal brachiocephalic trunk

- Lesions of the thoracic aorta

- Lesions of the abdominal aorta

Abbreviation: ESR, erythrocyte sedimentation rate.

has a sensitivity of $90.5 \%$ and a specificity of $97.8 \%$ for diagnosis of TA. These criteria are not diagnostic but rather a design to distinguish patients with TA from patients with other forms of vasculitis. With the Ishikawa criteria, in addition to the presence of the obligatory criterion, the presence of 2 major plus 4 minor or 1 major plus 2 minor criteria suggests a high probability of Takayasu's disease with $84 \%$ sensitivity.

\section{Diagnostic Criteria Giant Cell Arteritis}

The diagnosis of GCA is still largely a clinical diagnosis with confirmation by vessel biopsy. As listed in - Table 3, the diagnostic criteria issued by the American College of Rheumatology in 1990 are still widely used. The presence of 3 or more criteria yields a diagnostic sensitivity of $93.5 \%$ and specificity of $91.2 \%$.

\section{Prognosis of Takayasu's Arteritis}

The natural disease progression of TA has come from mainly one longitudinal study conducted in Japan. Ishikawa and Maetani, the researchers of this study, developed a prognostic classification of TA based on a cohort of 120 patients. According to the authors, there are four significant predictors that determine long-term survival in patients with TA. Those factors are major complications of vascular pathology (i.e., Takayasu's retinopathy, hypertension, aortic regurgitation, and aneurysm), progressive course of symptoms and pathology, age of the patient, and the calendar year of diagnosis. Based on all of these factors, the average survival rate was $82.9 \%$ of the cohort after 15 years. Although the authors found that elevated ESR was marginally significant, a high

Table 1 American College of Rheumatology 1990 criteria for the classification of Takayasu's arteritis

- Age at onset of disease less than or equal to 40 years.

- Claudication of extremities: development and worsening of fatigue and discomfort in muscles of 1 or more extremity while in use.

- Decreased brachial artery pulse.

- Systolic BP difference greater than $10 \mathrm{~mm} \mathrm{Hg}$ between each arm.

- Bruit over subclavian arteries or abdominal aorta.

- Arteriographic abnormality: narrowing or occlusion of the entire aorta, its primary branches or large arteries in the proximal upper or lower extremities, not due to arteriosclerosis, fibromuscular dysplasia, or similar causes; changes usually focal or segmental. 
Table 3 The American College of Rheumatology [ACR] classification criteria for GCA

The American College of Rheumatology (ACR) classification criteria for GCA:

(i) Age at disease onset $>55$ years: development of symptoms or findings beginning at the age $>55$ years.

(ii) New headache: new onset of or new type of localized pain in the head.

(iii) Temporal artery abnormality: temporal artery tenderness to palpation or decreased pulsation, unrelated to arteriosclerosis of cervical arteries.

(iv) Elevated ESR: ESR550 mm/h by the Westergren method.

(v) Abnormal artery biopsy: biopsy specimen with artery showing vasculitis characterized by a predominance of mononuclear cell infiltration or granulomatous inflammation, usually with multinucleated giant cells.

For purposes of classification, a patient shall be said to have GCA (TA) if at least three of these five criteria are present. The presence of any three or more criteria yields a sensitivity of $93.5 \%$ and a specificity of $91.2 \%$.

ESR was determined to be an independent predictor in a multivariate analysis, along with major complications of TA and progressive course. ${ }^{35}$

Ishikawa and Maetani used the independent predictors of survival, high ESR, major complications, and course progression to classify TA into three stages (I, II, and III). Stage I referred to TA without manifestations, and conferred the best prognosis with a low mortality rate. Meanwhile, stage III describes TA with major complications and progressive course and is associated with the worst prognosis with less than half of patients surviving after 15 years.

Therefore, prognosis was determined to be predicted by the presence/absence of major complications, a progressive course, and an elevated erythrocyte sedimentation rate. The authors of this study suggested aggressive medical and surgical treatment for patients in stage III; however, only $11.7 \%$ of the cohort were treated surgically between 1957 and $1990 .^{35}$ Currently, the mainstay of treatment of the active, inflammatory phase of TA is glucocorticoid therapy. Surgical intervention is necessary to bypass severe stenosis or occluded vessels.

\section{Prognosis of Giant Cell Arteritis}

Blindness is one of the most feared outcomes of GCA. Vision loss can be either partial or complete, and usually cannot be reversed. Without corticosteroid treatment, vision loss has been shown to occur between one- to two-thirds of patients. In almost half of patients with GCA, those who present with transient vision loss herald permanent vision loss. ${ }^{36}$ According to Danesh-Meyer et al, the first 6 days of the disease is when the patient is at the greatest risk of visual deterioration. Even if treatment with high-dose IV methylprednisolone is initiated, vision loss still occurs in $\sim 27 \%$ of patients. ${ }^{37}$

While GCA classically predominates in the temporal and cranial arteries, other large vessel manifestations are documented. Research suggests that patients with GCA were 17.3 times more likely to develop a thoracic aortic aneurysm than the general population. ${ }^{24}$ Aortic dissection is far more likely in patients with TAA. Consequently, GCA patients who develop structural damage to the aorta and associated vessels require surgical intervention. Apart from necessitating endovascular surgery as a result of aortic injury, patients with high-grade vascular stenosis in other locations may require limb amputation or endovascular surgery to avoid limb ischemia. ${ }^{38}$
Ultimately, the need for surgical intervention due to the vascular effects of GCA is very similar to the role of surgery in TA. Surgical interventions are discussed at length later in this review.

\section{Imaging in Vasculitis}

Various imaging techniques including high resolution ultrasound, magnetic resonance imaging (MRI), magnetic resonance angiography (MRA), computed tomography (CT) angiography, and positron-emission tomography (PET) scanning have been evaluated for the improved diagnosis and detection of active inflammation in LVV, as well as disease monitoring and surgical planning. Currently, there is no modality of choice in imaging for LVV, but PET scanning is evolving as a technique that can be used as both a diagnostic and prognostic tool. ${ }^{39}$ It provides a highly sensitive test, particularly in the early phase, and maintains the ability to localize disease activity with assessment of vascular morphology. CT and MRA are also useful methods in demonstrating stenosis, and providing accurate measurements in aneurysmal disease. Combined PET and CT scanners have eliminated the need for two separate imaging techniques. Furthermore, it allows for more precise anatomical location of disease activity and has been shown to be more sensitive in early diagnosis of LVV. The first trial to specifically evaluate the PET scan in large vessel vasculitis was performed in 1999, and, since then, several other authors have also published on this topic, all of which are retrospective in nature with the largest containing 46 patients. $^{40-42}$

\section{Imaging in Takayasu's Arteritis and Giant Cell Arteritis}

The best imaging in Takayasu's arteritis is highly debated; a gold standard imaging modality does not yet exist. In 2009, the European League against Rheumatism (EULAR) published recommendations for the management of LVV, suggesting both clinical and imaging assessment of the arterial tree when TA is suspected. ${ }^{43}$ Previously, the imaging modality of choice, catheter-based angiography fell out of favor due to its invasiveness. Consequently, CT and MRI began to supersede it because of the associated limitations, including arterial puncture, large doses of contrast and substantial radiation, and difficulty to perform in patients with heavy vessel calcifications or long segments of stenosis. ${ }^{44}$ Furthermore, angiography may produce a false positive in patients with early phase TA as changes in the arterial wall cannot be 
assessed with that modality. In addition to the known limitations of angiography, research suggests that angiography may increase the risk of ischemic complications, thought to arise from preexisting thrombus formation and subsequent embolism in TA. ${ }^{45,46}$ Despite these known complications, the use of angiography in interventional procedures, including endovascular stent deployment and angioplasty in the management of stenotic lesions, remains to be a commonly used modality.

Both polymyalgia rheumatica and GCA are clinical diagnoses with laboratory tests and temporal artery biopsies functioning as supporting but not definitive data. GCA traditionally required a temporal artery biopsy as a cornerstone of diagnosis. This practice is extremely limiting; in that it is time-consuming and is accompanied by a high falsenegative rate.

The Royal College of Physicians recommend that in the presence of a negative temporal artery biopsy treatment for GCA should still be commenced with clinical suspicion and a laboratory picture of GCA. ${ }^{47}$ Thus, similar to TA, GCA still suffers from the absence of a gold standard diagnostic test.

LVV occurs in $25 \%$ of GCA patients. ${ }^{48}$ Thus, investigation of the large vessels is necessary in patients with biopsy-confirmed GCA. MRA and CT angiography (CTA) help determine the extent of arterial involvement and disease progression. MRA and CTA have also been used to find large vessel involvement in patients without biopsy confirmation but with a high clinical suspicion of GCA and peripheral claudication. While not highly specific for inflammation, CTA and MRA do demonstrate delayed enhancement of the arterial wall due to intramural leaky microvessels. Thus, traditional angiography is less frequently used and is reserved for revascularization procedures rather than diagnosis. ${ }^{49}$

While the diagnostic imaging for GCA is yet to be determined, the importance of initial vessel evaluation, whether by CTA or MRA, cannot be underestimated. The 2010 AHA Aortic guidelines suggest that the initial evaluation of patients with confirmed GCA should undergo imaging of the thoracic aorta and its branches through either CT or MRI. ${ }^{50}$ However, a recent systematic review by Mackie et al warn that the true relative risk and the time course of the risk of developing thoracic aortic aneurysm in GCA is still unclear and before imaging clinicians should consider whether, and how, detecting aortic pathology would improve patient management. ${ }^{51}$ On the other hand, there is good evidence to support excellent outcomes in specialist aortic centers performing thoracic aortic surgical repair in octogenarians. ${ }^{52}$

\section{Use of CT Scan, MRI, and MRA in Patients with Large-Vessel Vasculitides}

CT scanning allows for the evaluation of luminal diameter, as well as arterial wall thickness, which is a pathological early feature of TA. However, CT scans still necessitate the exposure of high levels of radiation and the use of intravenous contrast. Furthermore, it is difficult to differentiate the aortic calcification exhibited in TA from that in long-standing atherosclerosis, particularly when long segment stenosis is not present.
MRI/MRA scanning is a useful imaging modality for TA in that it does not utilize ionizing radiation and can be used safely for serial imaging in the follow up and assessment of treatment in patients. In a study of $16 \mathrm{TA}$ patients performed by Tso et al, serial scans detected 12 new anatomic abnormalities in half of the participants ( 4 occlusions, 7 stenoses, and 1 aneurysm). ${ }^{53}$ With regards to diagnostic accuracy, the sensitivity and specificity of MRA are both $100 \%$ in TA. ${ }^{54}$ MRI can also be used to follow disease activity, showing vessel wall edema in $56 \%$ of patients thought to have been in clinical remission. However, this finding on imaging did not correlate to any changes in clinical presentation as 6 of 16 patients had no disease progression despite persistent vessel wall edema on MRI. ${ }^{39}$ Interestingly, the presence of vessel wall edema, thought to show active inflammation, was not necessary for the development of new lesions. MRI with high-field strength is also being investigated as a sensitive method to detect temporal artery inflammation. However, it is still far from replacing the gold standard of biopsy, which is highly sensitive to even minor inflammatory changes. ${ }^{49}$

MRI scanning offers similar findings as with CT scanning, as well as the possibility of detecting cardiovascular functional and hemodynamic changes. Further, MRI scanning is poor at visualizing small branch vessels and vascular calcification. Other limitations include its expense, time demand, and operator dependency. ${ }^{53}$

\section{Use of Combined PET-CT in LVV}

In comparison to MRI, the combined modality of 18F-FDGPET-CT has been shown to have high sensitivity and specificity in the early diagnosis of TA. In addition to its use in early-stage TA, 18F-FDG-PET-CT is better equipped to assess the degree and site of inflammation. ${ }^{42}$ In practice, however, diagnosis of early TA is very uncommon, presumably due to its benign clinical presentation with no defining features and rarity of incidence. ${ }^{55}$ Thus, in clinical practice, 18 F-FDG-PETCT in comparison to MRI show no significant difference in its sensitivity and specificity to diagnose TA ${ }^{56}$ However, 18 FFDG-PET-CT has been proven to identify more affected anatomic regions than those found in MRI.

Unlike in GCA, 18F-FDG-PET-CT has been shown in TA to be more useful in the follow-up of patients. ${ }^{57}$ Tezuka et al studied 39 Japanese TA patients who underwent serial 18FFDG-PET-CT imaging. The study demonstrated that the conventional method of ESR and CRP screening underestimates TA activity, whereas 18F-FDG-PET-CT showed a stronger correlation with clinical symptoms, including relapses during treatment. 18F-FDG-PET-CT also has the ability to detect hypermetabolic cells and signal inflammatory changes that may not yet be clinically active, with high sensitivity, thereby allowing it to predict outcomes well, making 18F-FDG-PET-CT a possible option for patient follow-up. ${ }^{42}$ On the other hand, the sensitivity and specificity of 18F-FDG-PET-CT is still contested as it is yet to be established in the literature. Thus, its routine use is still not recommended. ${ }^{49}$

Other shortcomings of 18F-FDG-PET-CT are that the patient is exposed to high levels of radiation, 15 to $20 \mathrm{mSv}$ 
for each scan, from both the $\mathrm{CT}$ and the radioisotope. ${ }^{41}$ In comparison, a chest X-ray equates to $\sim 0.02 \mathrm{mSv}$ and a CT thorax $6.6 \mathrm{mSv}$. Furthermore, there are no standardized measurements for quantification of FDG uptake, which makes clinical comparisons difficult to assess. ${ }^{58}$ Clinically inactive disease with normal acute phase reactants may still show FDG uptake, and clinically active disease may suppress FDG uptake.

As mentioned above, the role of 18F-FDG PET in GCA scanning is still debated. The lack of a defined protocol, questionable accuracy, and its exact clinical role in diagnosis management and follow-up all remain unanswered questions. As with TA, one main limitation is that reporting of a scan relies on semiquantitative analysis of uptake. ${ }^{59}$ Furthermore, this can vary depending on when the scan is taken after administration of 18F-FDG, and whether the patient fasted before the scan. Increasingly, combined 18 F-FDG-PET-CT scanners are being used, with the coregistered images allowing more precise anatomic location of metabolic activity, thereby enhancing the test's sensitivity, particularly in the event of moderate FDG accumulation. ${ }^{60}$

The current literature demonstrates that 18F-FDG PET scanning is not consistent in its ability to detect GCA. Sensitivity and specificity range from $56 \%$ to $100 \%$ and $72 \%$ to $100 \%$, respectively, have been reported. ${ }^{46,47,61}$ Some of this variability is because patients already established on corticosteroid treatment do not display good uptake of $18 \mathrm{~F}-$ FDG. A study of 78 patients in 2011 showed that $18 \mathrm{~F}$-FDG PET scanning was unreliable in confirming the diagnosis of vasculitis in patients already sustained on steroid therapy. ${ }^{62}$ In GCA, corticosteroids are initiated very early on to ensure eyesight is preserved. Coupled with no defined use as a diagnostic tool in GCA, PET scanning is rarely used before initiation of corticosteroids. This has been reinforced by numerous case reports describing the use of 18F-FDG PET scanning to diagnose GCA. ${ }^{63-65}$

PET scanning, in comparison to MRI, has shown comparable sensitivities in diagnosing. However, PET scanning allows simultaneous identification of more affected vessels than MRI.

18F-FDG PET scanning may warrant use as a surrogate marker of disease activity during follow-up. It monitors disease activity noninvasively and allows for assessment of immunosuppressive therapy. Finally, when used in this early stage, 18F-FDG PET scanning is an excellent tool for measuring the extent of active inflammation in large vessel vasculitis. This is of particular interest when considering aortic surgery. Suggested screening for aneurysms in patients with GCA includes baseline and annual abdominal ultrasound, chest radiography, and transthoracic echocardiography, although these cannot accurately assess the thoracic aorta.

Despite much debate to identify the gold standard investigation for diagnosis and follow-up, a consensus is yet to be found for the best imaging modality in the follow-up of patients. The various imaging modalities discussed above can, however, provide a good insight into healthy, nonaffected tissue, and thus give insight to the planning of surgical repair for sites of anastomoses.

\section{Management and Outcomes of Takayasu's Arteritis and Giant Cell Arteritis}

The management of these, often complex, patients with vasculitis is with an experienced multidisciplinary team (MDT), including surgeons, radiologists, and physicians. Typically, the long-term complications of LVV such as aneurysm formation, vessel stenosis, or valvular pathology are managed regardless of their etiology. Treatment is often based on size criteria and the patient's symptoms with the aim being for prognostic or symptomatic benefit.

It was only until the invention of cardiopulmonary bypass that repair of the thoracic aorta could be considered in the majority of patients. Thoracic aortic surgery is, therefore, a relatively new specialty. These are covered extensively in the AHA guidelines on thoracic aortic disease.

Acute presentations of the vasculitides, such as aortic dissection or rupture, are managed with previously welldefined interventions and require either surgical or endovascular intervention.

Preoperatively, close interrogation of the CT-PET should take place. It is not unreasonable to identify the extent of the inflammatory process with the aim of avoiding anastomotic strictures at active sites. In addition, within the limits of safety, resection should be as extensive as possible. Other techniques include the use of Teflon strips or patches to reinforce anastomotic sites, and postoperative corticosteroids to suppress further inflammation. ${ }^{66}$

Follow-up is mandatory with input again from the MDT. Surveillance for ongoing or recurrent disease and its complications should involve biomarkers, imaging, and clinical suspicion. These patients may require lifelong treatment and intervention necessitating referral to specialist centers, particularly when complex scenarios, such as acute, active vasculitis or graft infection, are encountered. ${ }^{67}$

The dilemmas regarding the surgical management of vasculitis focus on subacute processes when there is active disease with surgically relevant consequences. Timing of surgery in this setting is key to a successful outcome.

\section{Thoracic Aortic Management of Takayasu's Arteritis}

\section{Surgical Management}

Surgical management of TA largely depends upon the progression of the disease into the aorta and the clinical status. Aortic involvement is quite common in these patients, with aneurysms reported in up to $40 \%$. Interventions for such cases are largely depending on clinical presentation and the progression of dilatation of the involved aorta. The interventions are approached through percutaneous stenting but this is associated with risk of further dilatation of the native aorta, stent migration, endoleaks, and stenosis, ${ }^{68,69}$ while the definite management is through open surgical replacement of the diseased aorta. Yet, the open repair is also has its own limitation especially in the presence of active disease which has a requirement for a revision surgery at later stage. It is reported that 5 and 10 -year freedom from revision was 
$57 \%$ in patients with active disease on steroids compared with $100 \%$ in patients with quiescent disease not requiring steroids. $^{70}$

\section{Medical Management}

Despite medical treatment, TA is well known to be a detrimental disease process with substantial morbidity. The burden of TA was demonstrated in a retrospective study from the Cleveland clinic that studied 75 patients treated for TA between 1992 to $2004 .^{71}$ In their cohort of patients, 28 patients achieved remission during the study period, and of these, 27 had a disease relapse. Eighteen of 30 patients had vascular claudication that impaired the performance of routine daily activities and 23 patients were unable to work. Five of 30 patients (17\%) experienced cerebral ischemic disease ( 2 had transient ischemic attacks and 3 had strokes). During this study two patients died from disease-related deaths.

\section{Thoracic Aortic Management of Giant Cell Arteritis}

\section{Surgical Management}

In addition to affecting the temporal and cranial arteries, GCA can impact other large vessels including the thoracic aorta, although it is rare.

Lie et al, in an assessment of 72 patients with aortic or extra-cranial GCA, reported that the ascending and arch of the aorta were the most commonly involved, with $39 \%$ of the patients being affected. ${ }^{72} 12$ patients suffered fatalities directly attributed to aortic aneurysm or dissection. Furthermore, Evans et al described 18 patients out of a 41-patient cohort with GCA and a known TAA that required over 20 surgical procedures. ${ }^{24}$ In the same study, 16 patients developed dissection, half of which died as a result. Aortic insufficiency was detected in 19 patients as a consequence of aortic root dilation.

\section{Medical Management}

As mentioned above, aortic dissection can occur even without the presence of a previously documented aneurysm. This suggests that aortic inflammation, regardless of its presence in the course of the disease, may be a component of GCA that predisposes patients toward dissection. Should this be the pathogenesis of GCA dissection, persistent aortitis could be identified and treated with steroid therapy before dissection. This holds true in the literature in that dissection in GCA tends to occur during the early onset of the disease, which is attributed to inadequate disease suppression. We must try to identify those patients at risk of aortitis and therefore dissection. 18 F-FDG-PET-CT scanning could be the ideal modality to assess the aorta; however, the risk of frequent high-dose radiation still outweighs the minimal benefits of the information obtained. Many centers still advocate the use of 2-view chest radiograph despite the low sensitivity of chest radiography. A recent systematic review described that on average 5 to 10 patients with GCA would need aortic cross sectional imaging to detect one previously unknown thoracic aortic aneurysms or dissection. ${ }^{44}$
No guidelines exist as to whether these patients should be prescribed steroids pre- or postoperatively; however, experiences by the authors suggest this produces better outcomes.

Fields et al report that surgical intervention in 42 patients with active disease increased the rate of revision or development of progressive symptomatic disease at another site. $^{55}$

\section{A Brief Word on Immunoglobulin G4-Related Disease}

Immunoglobulin G4-related disease [Igg4-RD], is a rare systemic fibroinflammatory disorder that can affect any organ. While type 1 autoimmune pancreatitis is the most frequent manifestation of IgG4-RD, IgG4-RD can affect any organ or organ system, such as salivary glands, orbits, retroperitoneum, and many others. Of particular interest, IgG4$\mathrm{RD}$ is recognized as another cause of noninfectious aortitis in less than $5 \%$ of cases, apart from TA or GCA. Uchida et al described that the prevalence of this disease in Japan in 2009 was $\sim 8,000$ patients (60 per million inhabitants) with a slight male predominance. ${ }^{73}$ A Japanese report from a cohort of 125 patients show that $4 \%$ of patients with TAA could be attributed to IgG4-RD by histology. ${ }^{74}$ A German study of 376 patients who underwent resection of the thoracic aorta found evidence of inflammatory aortic aneurysms in $4 \%$ of patients. ${ }^{75}$ In these patients, there were highly overlapping histological features with IgG4-RD in the resected aorta, despite many patients lacking other systemic features associated with IgG4-RD and normal serum IgG4 levels. Whether this represents part of the spectrum of IgG4-RD or a separate pathological entity warrants further research.

Currently, treatment for this condition is completed with medical therapy, namely glucocorticoid therapy, although rituximab may hold potential future advances because of its ability to reduce circulating IgG4 levels. ${ }^{76}$ It is hard to draw conclusions about surgical management at this stage, because it is both rare and underdiagnosed.

Thus, physicians should be aware that IgG4-related disease accounts for a possible cause of aortitis and should still remain part of the differential diagnosis.

\section{Unanswered Questions}

The true prevalence of TA in many countries is still unknown, and the pathogenesis of thoracic aortic lesions in GCA patients is still undefined. As a consequence of this, there does not exist any guidelines for aortic monitoring. We must continue to seek the value of PET scanning and its use in the timing and planning of aortic intervention. Evaluating and monitoring patients for aortic dissection also remains to be an important unanswered question. Furthermore, when surgical intervention is undertaken for TAA, should noninfective aortitis be considered and revaluated via biopsy? If many studies of GCA and TA report the discovery of LVV at the time of diagnosis of TAA, then the diagnosis of LVV should be considered in patients with newly diagnosed TAA. A retrospective study in 2000 evaluated aortic surgical specimens gathered over a 20-year period from 
patients with TAA. Rojo-Leyva et al reported $12 \%$ of 383 patients with TAA demonstrated aortitis not attributable to previous surgery or atheroma, with the majority not displaying systemic symptoms of illness before the time of surgery. Furthermore, during a mean follow-up period of 41.2 months, new aneurysms were identified among 6 of 25 patients who were not treated with glucocorticoids. None were identified who were treated with glucocorticoids. Another question that still remains is how to best medically manage patients who undergo surgery on the thoracic aorta. In these cases, it is unclear whether the risks of immunosuppression outweigh the risk of restenosis.

Even if immunosuppression is used, it is still unclear whether active inflammation of the aorta actually increases the likelihood of postoperative complications.

\section{Summary}

While knowledge of large vessel vasculitis has existed for over a century, there have been few advances in diagnosis and surgical treatment of this condition apart from the development of aortic surgery. The rarity and heterogeneity of the disease have significantly contributed to the quality of published evidence, and thus, difficulty in determining the best clinical management.

Current research focuses on the increased need for disease awareness and early diagnosis, diagnostic imaging modalities, clinical follow-up of these patients, timing of surgical intervention, and medical treatment. Exciting evidence has been directed toward positron emitting scans as a method for assessing the extent and activity of these two diseases. Furthermore, this technique has been used in the prediction of outcomes preceding aneurysm repair and may have a role in planning of surgical repair. This hold clinical relevance in that patients undergoing aneurysm repair with LVV are subject to a high reoperation rate, notoriously from anastomotic aneurysm formation, compared with those with aneurysms not attributable to large vessel vasculitis. ${ }^{42,77}$ There has been little research to prevent this reoperation medically or surgically. Surgical intervention for LVV and the aorta is not a subject that has been thoroughly reviewed; current practice relies on a few published retrospective studies and case series, a majority originating from Japan. Answers could feasibly be obtained with multicenter retrospective analyses or cohorts, because prospective trials remain to be expensive, particularly considering the disease's rarity.

\section{Disclosures \\ None.}

\section{Conflict of Interest}

None.

\section{References}

1 Mason JC. Takayasu arteritis-advances in diagnosis and management. Nat Rev Rheumatol 2010;6(07):406-415

2 Takayasu M. A case with peculiar changes of the retinal central vessels. Acta Soc Ophthal Jpn. 1908;12:554-555
3 Shimizu K, Sano K. Pulseless disease. J Neuropathol Clin Neurol 1951;1(01):37-47

4 Numano F, Kakuta T. Takayasu arteritis-five doctors in the history of Takayasu arteritis. Int J Cardiol 1996;54(Suppl):S1-S10

5 Mason JC. Takayasu arteritis: surgical interventions. Curr Opin Rheumatol 2015;27(01):45-52

6 Ueno A, Awane Y, Wakabayashi A, Shimizu K. Successfully operated obliterative brachiocephalic arteritis (Takayasu) associated with the elongated coarctation. Jpn Heart J 1967;8(05):538-544

7 Hellman DB. Giant cell arteritis, polymyalgia rheumatica, and Takayasu's arteritis. In: Firestein GS, Budd RC, eds. Kelley's Textbook of Rheumatology, 9th ed. Philadelphia, PA: Elsevier Saunders; 2013:1461-1480

8 Sano K, Saito I. Pulseless disease. Summary of our experiences and studies. Schweiz Arch Neurol Neurochir Psychiatr 1972;111(02): 417-433

9 Ross RS, McKUSICK VA. Aortic arch syndromes; diminished or absent pulses in arteries arising from arch of aorta. AMA Arch Intern Med 1953;92(05):701-740

10 Salvarani C, Cantini F, Boiardi L, Hunder GG. Polymyalgia rheumatica and giant-cell arteritis. N Engl J Med 2002;347(04):261-271

11 Weyand CM, Goronzy JJ. Giant-cell arteritis and polymyalgia rheumatica. Ann Intern Med 2003;139(06):505-515

12 Rahman W, Rahman FZ. Giant cell (temporal) arteritis: an overview and update. Surv Ophthalmol 2005;50(05):415-428

13 Koide K. Takayasu arteritis in Japan. Heart Vessels Suppl 1992; 7:48-54

14 Watts R, Al-Taiar A, Mooney J, Scott D, Macgregor A. The epidemiology of Takayasu arteritis in the UK. Rheumatology (Oxford) 2009;48(08):1008-1011

15 Waern AU, Andersson P, Hemmingsson A. Takayasu's arteritis: a hospital-region based study on occurrence, treatment and prognosis. Angiology 1983;34(05):311-320

16 Reinhold-Keller E, Herlyn K, Wagner-Bastmeyer R, Gross WL. Stable incidence of primary systemic vasculitides over five years: results from the German vasculitis register. Arthritis Rheum 2005;53(01):93-99

17 Hall S, Barr W, Lie JT, Stanson AW, Kazmier FJ, Hunder GG. Takayasu arteritis. A study of 32 North American patients. Medicine (Baltimore) 1985;64(02):89-99

18 Koide K. Takayasu arteritis in Japan. Heart Vessels Suppl 1992;7 (Suppl.):48-54

19 Kerr GS, Hallahan CW, Giordano J, et al. Takayasu arteritis. Ann Intern Med 1994;120(11):919-929

20 Gulati A, Bagga A. Large vessel vasculitis. Pediatr Nephrol 2010;25 (06):1037-1048

21 Hutchinson J. Diseases of the arteries. On a peculiar form of thrombotic arteritis of the aged which is sometimes productive of gangrene. Arch Surg 1890;1:323-329

22 Goodwin JS. Progress in gerontology: polymyalgia rheumatica and temporal arteritis. J Am Geriatr Soc 1992;40(05):515-525

23 Kobayashi S, Fujimoto S. Epidemiology of vasculitides: differences between Japan, Europe and North America. Clin Exp Nephrol 2013;17(05):611-614

24 Nordborg E, Nordborg C. Giant cell arteritis: epidemiological clues to its pathogenesis and an update on its treatment. Rheumatology (Oxford) 2003;42(03):413-421

25 Liu NH, Labree LD, Feldon SE, et al. The epidemiology of giant cell arteritis: a 12-year retrospective study. Ophthalmology 2001;108 (06):1145-1149

26 Evans JM, O'Fallon WM, Hunder GG. Increased incidence of aortic aneurysm and dissection in giant cell (temporal) arteritis. A population-based study. Ann Intern Med 1995;122(07):502-507

27 Scheurer RA, Harrison AR, Lee MS. Treatment of vision loss in giant cell arteritis. Curr Treat Options Neurol 2012;14(01):84-92

28 Harky A, Fok M, Balmforth D, Bashir M. Pathogenesis of large vessel vasculitis: Implications for disease classification and future therapies. Vasc Med 2019;24(01):79-88 
29 Weyand CM, Goronzy JJ. Medium- and large-vessel vasculitis. N Engl J Med 2003;349(02):160-169

30 Nordborg E, Bengtsson BA, Nordborg C. Temporal artery morphology and morphometry in giant cell arteritis. APMIS 1991;99 (11):1013-1023

31 Weyand CM, Tetzlaff N, Björnsson J, Brack A, Younge B, Goronzy JJ. Disease patterns and tissue cytokine profiles in giant cell arteritis. Arthritis Rheum 1997;40(01):19-26

32 Goronzy JJ, Weyand CM. Cytokines in giant-cell arteritis. Cleve Clin J Med 2002;69(Suppl 2):SII91-SII94

33 O'Neill L, Molloy ES. The role of toll like receptors in giant cell arteritis. Rheum 2016;55(11):1921-1931

34 Sharma BK, Jain S, Suri S, Numano F. Diagnostic criteria for Takayasu arteritis. Int J Cardiol 1996;54(Suppl):S141-S147

35 Ishikawa K, Maetani S. Long-term outcome for 120 Japanese patients with Takayasu's disease. Clinical and statistical analyses of related prognostic factors. Circulation 1994;90(04):1855-1860

36 González-Gay MA, Blanco R, Rodríguez-Valverde V, et al. Permanent visual loss and cerebrovascular accidents in giant cell arteritis: predictors and response to treatment. Arthritis Rheum 1998;41(08):1497-1504

37 Danesh-Meyer H, Savino PJ, Gamble GG. Poor prognosis of visual outcome after visual loss from giant cell arteritis. Ophthalmology 2005;112(06):1098-1103

38 de Franciscis S, Roscitano G, Serra R, et al. Combined medical, surgical and endovascular treatment of a giant cell arteritis case manifesting as upper limbs acute ischemia. Int J Surg Case Rep 2011;2(05):71-73. Doi: 10.1016/j.ijscr.2011.02.003

39 Selvaraj A, Francis N, Harky A, et al. Imaging modalities in the management of aortitis. Indian J Thorac Cardiovasc Surg 2018. DOI: https://doi.org/10.1007/s12055-018-0710-7

40 Meller J, Grabbe E, Becker W, Vosshenrich R. Value of F-18 FDG hybrid camera PET and MRI in early takayasu aortitis. Eur Radiol 2003;13(02):400-405

41 Hara M, Goodman PC, Leder RA. FDG-PET finding in early-phase Takayasu arteritis. J Comput Assist Tomogr 1999;23(01):16-18

42 Webb M, Chambers A, AL-Nahhas A, et al. The role of 18F-FDG PET in characterising disease activity in Takayasu arteritis. Eur J Nucl Med Mol Imaging 2004;31(05):627-634

43 Mukhtyar C, Guillevin L, Cid MC, et al; European Vasculitis Study Group. EULAR recommendations for the management of large vessel vasculitis. Ann Rheum Dis 2009;68(03):318-323

44 Park JH, Chung JW, Lee KW, Park YB, Han MC. CT angiography of Takayasu arteritis: comparison with conventional angiography. J Vasc Interv Radiol 1997;8(03):393-400

45 Numano F. Pulmonary changes in Takayasu's arteritis. Junkanki Naika 1979;6:97-108

46 Akazawa H, Ikeda U, Yamamoto K, Kuroda T, Shimada K. Hypercoagulable state in patients with Takayasu's arteritis. Thromb Haemost 1996;75(05):712-716

47 Dasgupta B; Giant Cell Arteritis Guideline Development Group. Concise guidance: diagnosis and management of giant cell arteritis. Clin Med (Lond) 2010;10(04):381-386

48 Kermani TA, Warrington KJ, Crowson CS, et al. Large-vessel involvement in giant cell arteritis: a population-based cohort study of the incidence-trends and prognosis. Ann Rheum Dis 2013;72(12):1989-1994

49 Weyand CM, Goronzy JJ. Clinical practice. Giant-cell arteritis and polymyalgia rheumatica. N Engl J Med 2014;371(01):50-57

50 Hiratzka LF, Bakris GL, Beckman JA, et al; American College of Cardiology Foundation; American Heart Association Task Force on Practice Guidelines; American Association for Thoracic Surgery; American College of Radiology; American Stroke Association; Society of Cardiovascular Anesthesiologists; Society for Cardiovascular Angiography and Interventions; Society of Interventional Radiology; Society of Thoracic Surgeons; Society for Vascular Medicine. 2010 ACCF/AHA/AATS/ACR/ASA/SCA/SCAI/SIR/STS/SVM guidelines for the diagnosis and management of patients with thoracic aortic disease: executive summary-a report of the American College of Cardiology Foundation/American Heart Association Task Force on practice guidelines, American Association for Thoracic Surgery, American College of Radiology. Catheter Cardiovasc Interv 2010;76(02):E43-E86

51 Mackie SL, Hensor EM, Morgan AW, Pease CT. Should I send my patient with previous giant cell arteritis for imaging of the thoracic aorta? A systematic literature review and meta-analysis. Ann Rheum Dis 2014;73(01):143-148

52 Shah PJ, Estrera AL, Miller CC III, et al. Analysis of ascending and transverse aortic arch repair in octogenarians. Ann Thorac Surg 2008;86(03):774-779

53 Tso E, Flamm SD, White RD, Schvartzman PR, Mascha E, Hoffman GS. Takayasu arteritis: utility and limitations of magnetic resonance imaging in diagnosis and treatment. Arthritis Rheum 2002; 46(06):1634-1642

54 Yamada I, Nakagawa T, Himeno Y, Kobayashi Y, Numano F, Shibuya H. Takayasu arteritis: diagnosis with breath-hold contrast-enhanced three-dimensional MR angiography.J Magn Reson Imaging 2000;11(05):481-487

55 Sharma BK, Jain S, Suri S, Numano F. Diagnostic criteria for Takayasu arteritis. Int J Cardiol 1996;54(Suppl):S141-S147

56 Lin L, Tsai C, Chen M, et al. PET in vascular diseases : applications of FDG PET/CT in vasculitis and therapeutic response. Ann Nucl Med Sci 2006;19:91-98

57 Tezuka D, Haraguchi G, Ishihara T, et al. Role of FDG PET-CT in Takayasu arteritis: sensitive detection of recurrences. JACC Cardiovasc Imaging 2012;5(04):422-429

58 Blockmans D, Maes A, Stroobants S, et al. New arguments for a vasculitic nature of polymyalgia rheumatica using positron emission tomography. Rheumatology (Oxford) 1999;38(05): 444-447

59 Hautzel H, Sander O, Heinzel A, Schneider M, Müller H-W. Assessment of large-vessel involvement in giant cell arteritis with 18F-FDG PET: introducing an ROC-analysis-based cutoff ratio. J Nucl Med 2008;49(07):1107-1113

60 Papathanasiou ND, Du Y, Menezes LJ, et al. 18F-Fludeoxyglucose $\mathrm{PET} / \mathrm{CT}$ in the evaluation of large-vessel vasculitis: diagnostic performance and correlation with clinical and laboratory parameters. Br J Radiol 2012;85(1014):e188-e194

61 Turlakow A, Yeung HW, Pui J, et al. Fludeoxyglucose positron emission tomography in the diagnosis of giant cell arteritis. Arch Intern Med 2001;161(07):1003-1007

62 Walter MA, Melzer RA, Graf M, Tyndall A, Müller-Brand J, Nitzsche EU. [18F]FDG-PET of giant-cell aortitis. Rheumatology (Oxford) 2005;44(05):690-691[Oxford]

63 Schäfer VS, Warrington KJ, Williamson EE, Kermani TA. Delayed diagnosis of biopsy-negative giant cell arteritis presenting as fever of unknown origin. J Gen Intern Med 2009;24(04): 532-536

64 Yamashita H, Kubota K, Takahashi Y, et al. Whole-body fluorodeoxyglucose positron emission tomography/computed tomography in patients with active polymyalgia rheumatica: evidence for distinctive bursitis and large-vessel vasculitis. Mod Rheumatol 2012;22(05):705-711

65 Prabhavalkar S, Bogusz P, Merard R, Grormley M. An Unusual Presentation of Giant Cell Arteritis. Case Rep Med 2012;2012:1-3

66 Erdogan A, Gilgil E, Oz N, Türk T, Demircan A. PTFE patching to prevent anastomotic aneurysm formation in Takayasu's arteritis. Eur J Vasc Endovasc Surg 2003;25(05):478-480

67 Balmforth D, Harky A, Adams B, Yap J, Shipolini A, Roberts N, Uppal $\mathrm{R}$, Bashir $\mathrm{M}$. Is there a role for biomarkers in thoracic aortic aneurysm disease? Gen Thorac Cardiovasc Surg 2019;67(01):12-19

68 Min PK, Park S, Jung JH, et al. Endovascular therapy combined with immunosuppressive treatment for occlusive arterial disease in patients with Takayasu's arteritis. J Endovasc Ther 2005;12(01): $28-34$ 
69 Qureshi MA, Martin Z, Greenberg RK. Endovascular management of patients with Takayasu arteritis: stents versus stent grafts. Semin Vasc Surg 2011;24(01):44-52

70 Fields CE, Bower TC, Cooper LT, et al. Takayasu's arteritis: operative results and influence of disease activity. J Vasc Surg 2006;43 (01):64-71

71 Maksimowicz-McKinnon K, Clark TM, Hoffman GS. Limitations of therapy and a guarded prognosis in an American cohort of Takayasu arteritis patients. Arthritis Rheum 2007;56(03):1000-1009

72 Lie JT. Aortic and extracranial large vessel giant cell arteritis: a review of 72 cases with histopathologic documentation. Semin Arthritis Rheum 1995;24(06):422-431

73 Uchida K, Masamune A, Shimosegawa T, Okazaki K. Prevalence of IgG4-related disease in Japan based on nationwide survey in 2009. Int J Rheumatol 2012;2012:358371
74 Kasashima S, Zen Y, Kawashima A, et al. A clinicopathologic study of immunoglobulin G4-related sclerosing disease of the thoracic aorta. J Vasc Surg 2010;52(06):1587-1595

75 Agaimy A, Weyand M, Strecker T. Inflammatory thoracic aortic aneurysm (lymphoplasmacytic thoracic aortitis): a 13year-experience at a German Heart Center with emphasis on possible role of IgG4. Int J Clin Exp Pathol 2013;6(09): 1713-1722

76 Pieringer H, Parzer I, Wöhrer A, Reis P, Oppl B, Zwerina J. IgG4related disease: an orphan disease with many faces. Orphanet J Rare Dis 2014;9:110

77 Trimarchi S, Nienaber CA, Rampoldi V, et al; IRAD Investigators. Role and results of surgery in acute type B aortic dissection: insights from the International Registry of Acute Aortic Dissection (IRAD). Circulation 2006;114(1, Suppl)I357-I364 